\title{
Selective Cochlear Degeneration in Mice Lacking the F-Box Protein, Fbx2, a Glycoprotein-Specific Ubiquitin Ligase Subunit
}

\author{
Rick F. Nelson, ${ }^{1,2,3}$ Kevin A. Glenn, ${ }^{4}$ Yuzhou Zhang, ${ }^{5}$ Hsiang Wen, ${ }^{3}$ Tina Knutson, ${ }^{3}$ Cynthia M. Gouvion, ${ }^{3}$ \\ Barbara K. Robinson, ${ }^{5}$ Zouping Zhou, ${ }^{3}$ Baoli Yang, ${ }^{3,6}$ Richard J. H. Smith,, 5 and Henry L. Paulson ${ }^{2,3,7}$ \\ ${ }^{1}$ Medical Scientist Training Program, ${ }^{2}$ Graduate Program in Neuroscience, Departments of ${ }^{3}$ Neurology, ${ }^{4}$ Internal Medicine, ${ }^{5}$ Otolaryngology-Head and \\ Neck Surgery, and ${ }^{6}$ Pediatrics, and ${ }^{7}$ Graduate Program in Genetics, University of Iowa Roy J. and Lucille A. Carver College of Medicine, Iowa City, Iowa \\ 52242
}

Little is known about the role of protein quality control in the inner ear. We now report selective cochlear degeneration in mice deficient in Fbx2, a ubiquitin ligase F-box protein with specificity for high-mannose glycoproteins (Yoshida et al., 2002). Originally described as a brain-enriched protein (Erhardt et al., 1998), Fbx2 is also highly expressed in the organ of Corti, in which it has been called organ of Corti protein 1 (Thalmann et al., 1997). Mice with targeted deletion of Fbxo2 develop age-related hearing loss beginning at 2 months. Cellular degeneration begins in the epithelial support cells of the organ of Corti and is accompanied by changes in cellular membrane integrity and early increases in connexin 26, a cochlear gap junction protein previously shown to interact with Fbx2 (Henzl et al., 2004). Progressive degeneration includes hair cells and the spiral ganglion, but the brain itself is spared despite widespread CNS expression of Fbx2. Cochlear Fbx2 binds Skp1, the common binding partner for F-box proteins, and is an unusually abundant inner ear protein. Whereas cochlear Skp1 levels fall in parallel with the loss of Fbx2, other components of the canonical SCF (Skp1, Cullin1, F-box, Rbx1) ubiquitin ligase complex remain unchanged and show little if any complex formation with Fbx2/Skp1, suggesting that cochlear Fbx2 and Skp1 form a novel, heterodimeric complex. Our findings demonstrate that components of protein quality control are essential for inner ear homeostasis and implicate Fbx2 and Skp1 as potential genetic modifiers in age-related hearing loss.

Key words: deafness; ubiquitin; Fbx2; glycoprotein; Skp1; degradation

\section{Introduction}

Regulated protein turnover is essential for cellular homeostasis (Sitia and Braakman, 2003). Misfolded or unassembled proteins are commonly targeted for destruction via the ubiquitin proteasome pathway, with target substrates being recognized by specific ubiquitin ligases. The specificity of substrate ubiquitination in the Skp1/Cullin1/F-Box/Rbx1 (SCF) ubiquitin ligase complex is provided by F-box-containing proteins.

Fbx2 (also called Fbg1, Fbs1, NFB42, and OCP1) is a brain enriched F-box protein that specifically binds high-mannose, asparagine $(\mathrm{N})$-linked glycoprotein substrates through its F-box associated (FBA) domain (see Fig. 1a) (Yoshida et al., 2002). High-mannose N-linked glycans, which are signature elements of proteins in the endoplasmic reticulum (ER), facilitate chaperone-

Received Jan. 17, 2007; revised April 2, 2007; accepted April 4, 2007.

This work was supported by National Institutes of Health (NIH) Predoctoral Training Grant F30 NS047872 (R.F.N.) and NIH Grants R01 NS047535 (H.L.P.), R01 DC002842 (R.J.H.S.), and R01 DC003544 (R.J.H.S.). We thank Adam Mallinger and Rachael Wilson for genotyping, Penny Harding for tissue preparation, Michael Henry and Kevin Campbell for pPNTfloxNeo vector, Guy Van Camp and laboratory for FBX02 SNP analysis, and the members of the Paulson and Smith laboratories for critical, helpful comments on this manuscript.

Correspondence should be addressed to Henry L. Paulson, Department of Neurology, University of lowa College of Medicine, 2007 RCP, lowa City, IA 52242. E-mail: henry-paulson@uiowa.edu.

DOI:10.1523/JNEUROSCI.0206-07.2007

Copyright $\odot 2007$ Society for Neuroscience $\quad$ 0270-6474/07/275163-09\$15.00/0 mediated folding of nascent glycoproteins in the ER and serve as recognition markers for degradation through ER-associated degradation (ERAD) (Helenius and Aebi, 2004; Meusser et al., 2005). $\mathrm{Fbx} 2$ is a cytoplasmic protein that binds high-mannose glycans and is believed to mediate the ubiquitination of ER glycoproteins destined for ERAD (Yoshida et al., 2002). Failures in ERAD lead to ER stress and disease in the nervous system and other organ systems (Schroder and Kaufman, 2005).

Fbx2 was initially reported to be expressed only in postnatal brain and testes (Erhardt et al., 1998; Yoshida et al., 2003). However, orthologs of Fbx2 and Skp1 had previously been identified in the guinea pig as abundant proteins in the organ of Corti, the sensory organ of the cochlea, and are called organ of Corti proteins 1 and 2 (OCP1 and OCP2), respectively (Thalmann et al., 1997). These two functionally linked ubiquitin ligase subunits display robust and overlapping expression in the cochlea (Thalmann et al., 1997), suggesting a connection between protein quality control and auditory function.

In the cochlea, mechanosensitive hair cells transduce mechanical waves to electrical signals (Corey et al., 2004) through potassium-mediated depolarization (Jentsch, 2000). The elevated endolymph potassium concentration, which is necessary for proper hair cell depolarization, is maintained by the surrounding epithelial support cell system of the organ of Corti (for 
review, see Jentsch, 2000). These cells are linked by gap junctions comprised of connexin protein subunits, predominantly connexin 26 (Cx26), which allow for rapid recycling of potassium and other small molecules (Beltramello et al., 2005). Mutations in Cx26 cause the most common form of genetic deafness in whites (Kelsell et al., 1997).

Gap junctions, including Cx26, are turned over rapidly (Berthoud et al., 2004) by both the proteasome (Laing and Beyer, 1995) and lysosome (Guan and Ruch, 1996). However, the cellular and molecular factors regulating $\mathrm{Cx} 26$ turnover and the in vivo functional consequences of this turnover are still unknown. Recently, Fbx2 was shown to coprecipitate Cx26 in the cochlea (Henzl et al., 2004), suggesting that this vital inner ear protein could be a substrate for Fbx2-mediated ubiquitination and degradation.

The reported interaction of $\mathrm{Fbx} 2$ with $\mathrm{Cx} 26$ further raises the possibility that Fbx2 regulates Cx26-dependent events in the cochlea. Here, we have explored these possibilities by generating and characterizing mice null for Fbx2, and we show that this F-box protein is important to inner ear homeostasis.

\section{Materials and Methods}

Generation of Fbxo2-1- mice. The Fbxo2 ${ }^{-1-}$ mice were generated by standard homologous recombination techniques. Using a probe derived from rat Fbxo2 cDNA, we screened a 129sv mouse bacterial artificial chromosome (BAC) genomic library (Research Genetics, Huntsville, $\mathrm{AL})$ to identify BAC clones containing the mouse $\mathrm{Fbxo} 2$ gene. Clone 544J-2, which contains the mouse Fbxo2 gene, was used as a source for genomic fragments to generate the targeting vector. The targeting plasmid (see Fig. $1 b$ ) was constructed in a modified pPNTfloxNeo vector (Williamson et al., 1997). A $2.8 \mathrm{~kb} \mathrm{BamHI}$ fragment immediately $5^{\prime}$ of exon 1 of the Fbxo2 gene was inserted into the BamHI cloning site of pPNTfloxNeo vector. The floxed Neo cassette replaced the first five exons and introns of Fbxo2. A $3.2 \mathrm{~kb} \mathrm{XhoI/EcoRV} \mathrm{fragment} \mathrm{of} \mathrm{Fbxo2} \mathrm{encom-}$ passing the sixth and final exon was subcloned into Bluescript and was inserted into the ClaI site of the pPNTfloxNeo vector. The Fbxo2 gene sequences flanking the neo cassette were confirmed by restriction enzyme digestion and sequencing.

The Fbxo2 targeting construct was linearized with Not I and electroporated into R1 embryonic stem (ES) cells. After double selection, ES cell clones were screened by Southern blot analysis to identify correctly targeted clones. Genomic DNA was digested with EcoRI, electrophoresed on agarose gels, transblotted to Hybond-N+ transfer membranes (GE Healthcare, Piscataway, NJ), and then hybridized at $65^{\circ} \mathrm{C}$ with a ${ }^{32} \mathrm{P}$ labeled, $0.7 \mathrm{~kb} E c o \mathrm{RI}-\mathrm{Bam} \mathrm{HI}$ probe that resides just $5^{\prime}$ of the targeted site in Fbxo2. This probe detects a wild-type (WT) band of $\sim 10 \mathrm{~kb}$ and a correctly targeted band of $3.5 \mathrm{~kb}$. Seven correctly targeted clones were identified in 296 analyzed clones from two separate electroporations of ES cells.

Two correctly targeted ES clones (AS167 and AS198) were microinjected into C57BL/ 6 blastocysts and transferred to C57BL/6 mice. Germline transmission to progeny was assessed by PCR analysis from genomic DNA and later confirmed by Southern blot analysis. For PCR genotyping, primers amplified 328 and $543 \mathrm{bp}$ fragments from the targeted and wild-type alleles, respectively. Tail DNA was used for PCR using the following conditions: (1) $94^{\circ} \mathrm{C}$ for $3 \mathrm{~min}$, (2) $94^{\circ} \mathrm{C}$ for $30 \mathrm{~s}$, (3) $58^{\circ} \mathrm{C}$ for $45 \mathrm{~s},(4) 68^{\circ} \mathrm{C}$ for $1 \mathrm{~min},(5)$ repeat steps $2-4$ for 30 cycles, and $(6) 68^{\circ} \mathrm{C}$ for $5 \mathrm{~min}$. Three primers were used: P1, CTCCTAGGATGGCTTTGGTTC; P2, GAGATCAGCAGCCTCTGTTC, and P3, GGATAGCACGTCAGCCTCAG.

Cochlear tissue preparation, analysis, and imaging. Mice were anesthetized by intraperitoneal injection of ketamine $(100 \mathrm{mg} / \mathrm{kg})$ and xylazine $(9 \mathrm{mg} / \mathrm{kg}$ ) and perfused with PBS plus protease inhibitors (Sigma, St. Louis, MO). For immunofluorescence (IF) and transmission electron microscopy (TEM) analysis, the mice were fixed by perfusion with $4 \%$ paraformaldehyde (PFA). Cochleae were isolated, fixed overnight in PFA, decalcified in $0.5 \%$ EDTA for $48 \mathrm{~h}$, and embedded in Spurs for morphological analysis and TEM or in paraffin for immunofluorescence. Cochlear sections were processed through the midmodiolus region of the cochlea. For Western blot analysis, mice were anesthetized and perfused with PBS plus protease inhibitors, after which the brain or cochleae were dissected and lysed in brain immunoprecipitation (IP) buffer $(20 \mathrm{~mm}$ HEPES, pH 7.4, $150 \mathrm{~mm} \mathrm{NaCl}, 1 \%$ Triton X-100, and 10\% glycerol) at $\sim 100 \mathrm{mg} / \mathrm{ml}$. Dissected cochleae (two per mouse) and a small residual amount of temporal bone were pulverized in $1.5 \mathrm{ml}$ Eppendorf (Hamburg, Germany) tubes containing $250 \mu \mathrm{l}$ of IP buffer. Equal amounts of protein were loaded after determining protein concentration by Bradford assay.

Immunofluorescence was visualized with an Axioplan fluorescence microscope (Zeiss, Thornwood, NY) using 20, 40, and $63 \times$ objectives. Images were captured digitally with a Zeiss MRM AxioCam camera and assembled in Photoshop 7.0 (Adobe Systems, Mountain View, CA). Fluorescence intensity was controlled using identical capture time between genotypes for each antibody $(\mathrm{Ab})$, and images were captured on a linear scale. Confocal imaging of immunofluorescent images was performed using the MRC-1024 confocal microscope (Bio-Rad, Hercules, CA) in conjunction with LaserSharp 2000 software. TEM images were acquired using the Gatan (Pleasanton, CA) UltraScan $10002000 \times 2000$ pixel CCD camera on the JEM 1230 (JEOL, Peabody, MA; University of Iowa).

Antibodies. Fbx2 was detected using a peptide rabbit polyclonal generated against amino acids 1-20 (MDGDGDPESVGQPEEASPEE), used at 1:2000 for Western blots (WBs) and 1:1000 for IF. Skp1 rabbit polyclonal Ab (s.c.-7163; Santa Cruz Biotechnology, Santa Cruz, CA) was used at 1:200 (WB) and 1:50 (IF), and Skp1 mouse monoclonal Ab (610530; BD Biosciences Pharmingen, San Diego, CA) was used at 1:5000 (WB). Ubiquitin was detected using the rabbit polyclonal Ab (Z0458; DakoCytomation) at 1:1000 (WB) and 1:100 (IF). Rbx1/Roc1, Cullin1, and Cullin7 were detected using rabbit polyclonal Abs at 1:100 (WB; RB-069; NeoMarkers, Fremont, CA), at 1:250 (WB; 71-8700; Zymed, San Francisco, CA), and at 1:500 (WB; BL-1541; Bethyl, Montgomery, TX), respectively. The tubulin mouse monoclonal Ab was used at 1:20,000 (WB; clone B-5-1-2, T5168; Sigma). The mouse glyceraldehyde-3-phosphate dehydrogenase antibody (MAB374, Millipore) was used at 1:300. The ER chaperone calnexin was detected at 1:250 for WB and 1:50 for IF using rabbit polyclonal antibody (SPA-860; Stressgen Biotechnologies, San Diego, CA). The mouse monoclonal Cx26 (33-5800; Zymed) and rabbit polyclonal connexin 30 (71-2200; Zymed) Abs were used at 1:500 for WB and 1:50 for IF. The mouse monoclonal NR1 Ab was used at 1:250 for WB (556308; BD Biosciences Pharmingen). Anti-FLAG coimmunoprecipitations were performed with EZ-view Red Anti-FLAG M2 Affinity Gel (F2426; Sigma), and the FLAG epitope was detected on WB using either mouse monoclonal Ab (F4042; Sigma) at 1:2000 or rabbit polyclonal Ab (F7425; Sigma) at 1:1000 dilution. Secondary antibodies for IF (Alexa Fluor 568 and A11011) were from Invitrogen (Eugene, OR) and were used at 1:500. Secondary antibodies for WB (115-035-003 and 111-035144) were from Jackson ImmunoResearch Laboratories (West Grove, PA) and were used at 1:15,000. High-mannose glycoproteins were visualized in cochlear sections with Oregon Green 488 ConA (C6741; 1:25; Invitrogen) and on $\mathrm{WB}$ with peroxidase-conjugated concanavalin $\mathrm{A}$ (ConA; Sigma) at $0.23 \mu \mathrm{g} / \mathrm{ml}$ TBS-Tween 20 dilution supplemented with $1 \mathrm{mM} \mathrm{CaCl}_{2}$ and $1 \mathrm{~mm} \mathrm{MnCl}_{2}$. TO-PRO-3 iodide (Invitrogen) was used to visualize nuclei in IF sections at 1:2000.

Auditory brainstem responses. After mice were anesthetized by intraperitoneal injection of ketamine $(100 \mathrm{mg} / \mathrm{kg})$ and xylazine $(9 \mathrm{mg} / \mathrm{kg})$, three stainless steel needle electrodes were placed at vertex as a reference, ventrolateral to right ear as active, and ventrolateral to left ear as ground. Body temperature was maintained at $37^{\circ} \mathrm{C}$ using a heating pad. Stimulus signals were generated by custom-designed software (Labview version 5 ). Biphasic click sounds (100 $\mu$ s/phase) with $31 \mathrm{~ms}$ interstimulus intervals were unilaterally presented at the right ear via a Beyer-DT-48 microphone inserted into the external auditory canal. Auditory brainstem responses (ABRs) were recorded in a double-walled sound-isolated sound booth. ABR signals were amplified $1000 \times$ using a DL Instruments (Ithaca, NY) model 1201 differential amplifier. Stimulus levels were gradually reduced by $10-20 \mathrm{~dB}$ and finally by $5 \mathrm{~dB}$ steps until no response was detected. Littermates were used for all cohort analyses. 
Quantification and statistical analysis. Western blot densitometry was performed using ImageJ software on exposures with band densities within the linear range. Statistical significance was determined using the Student's $t$ test.

Plasmids. Human FBXO2 cDNA was obtained from IMAGE clone 5090925, and human FBXO6 was cloned from heart and brain cDNA libraries, provided by Dr. Bento Soares (Northwestern Children's Memorial Hospital, Chicago, IL). Fbxo2 and Fbxo6 cDNAs ligated into pFLAG-CMV-6b (Sigma) at the HindIII/EcoRI and EcoRI/BglII sites, respectively. All cDNA clones used throughout this study were sequence verified. Human SKP1A in pcDNA3.0 and human N-terminal hemagglutinin-tagged CUL1 in pcDNA3.0 were gifts from Yue Xiong at the University of North Carolina Cancer Center (Chapel Hill, NC) (Michel and Xiong, 1998). Human RBX1 cDNA was inserted into the NotI/EcoRI site of pcDNA3.1(-).

Cell culture and transfections. Cos cells were grown in DMEM (Invitrogen) supplemented with 10\% fetal bovine serum (HyClone, Logan, UT) and $1 \%$ penicillin/streptomycin (Invitrogen) and were maintained at $37^{\circ} \mathrm{C}$ with $5 \% \mathrm{CO}_{2}$. Transfections were performed with Lipofectamine Plus reagent (Invitrogen) according to the manufacturer's protocol.

Immunoprecipitation. Endogenous $\mathrm{Fbx} 2$ was immunoprecipitated from $\sim 80 \mu \mathrm{g}$ of cochlear or brain lysate using the $\mathrm{Fbx} 2 \mathrm{Ab}$ and True-blot rabbit IgG beads (00-8800; eBioscience, San Diego, CA). After rotation at $4^{\circ} \mathrm{C}$ for $1 \mathrm{~h}$, beads were washed three times in $500 \mu \mathrm{l}$ of lysis buffer and eluted with $2 \times$ SDS plus $1 \mathrm{~mm}$ DTT. To denature IgG chains, precipitates were boiled for $10 \mathrm{~min}$ before analysis by SDS-PAGE. Western blots were performed with various antibodies listed above and detected using the anti-rabbit IgG secondary (18-8816; eBioscience) at 1:1000. Coprecipitation of FLAG-tagged proteins expressed in Cos cells was performed using EZ view anti-FLAG beads. All experiments were performed with cotransfection of Skp1, Cullin1, and Rbx1. Beads were prewashed twice with $1 \mathrm{ml}$ of lysis buffer before IP at $4^{\circ} \mathrm{C}$ for $1 \mathrm{~h}$. Beads were then washed four times with $1 \mathrm{ml}$ of lysis buffer and eluted with $2 \times$ SDS sample buffer without DTT and boiling to prevent dissociation of mouse IgG and detection by Western blot. Samples were then electrophoresed by SDSPAGE under reducing conditions. The 5\% input lane represents 5\% (in $\mu \mathrm{g}$ of protein) of the starting lysate subjected to immunoprecipitation.

Brain tissue analysis. Wild-type $\left(\mathrm{Fbxo2}^{+/+}\right), \mathrm{Fbxo2}^{+/-}$, and $\mathrm{Fbxo2} 2^{-1-}$ mice were anesthetized by intraperitoneal injection of ketamine (100 $\mathrm{mg} / \mathrm{kg}$ ) and xylazine $(9 \mathrm{mg} / \mathrm{kg})$, perfused with PBS plus protease inhibitors (Sigma), and perfusion fixed with $4 \%$ PFA. Brains were paraffin embedded and serially sectioned in the Pathology Tissue Core (University of Iowa) at $10 \mu \mathrm{m}$ intervals through the entire brain from the olfactory bulb through the brainstem. Parallel series of sections were histochemically stained with hematoxylin and eosin, Nissl, and Luxol Fast Blue and observed at $4 \times$ through $40 \times$ in an effort to detect global or regional differences in brain structure or integrity in adult $\mathrm{Fbxo2}^{-1-}$ mice. No overt histochemically detectable brain changes were observed (data not shown).

\section{Results}

To investigate the role of Fbx2 in vivo, we used homologous recombination to generate mice with a targeted deletion of the first five exons of the six-exon Fbxo2 gene $\left(\mathrm{Fbxo2}^{-1-}\right)$ (Fig. 1b). We confirmed this deletion by PCR (Fig. 1c), Western blot (Fig. 1d), and Southern blot analyses (data not shown). Backcrossing for nine generations placed the deletion on a C57BL/6J background. Obtained at the expected Mendelian ratios, $\mathrm{Fbxo2}^{-1-}$ mice are viable, fertile, and outwardly normal-appearing mice. They also display no circling or whirling behavior to suggest CNS or vestibular dysfunction. Fbxo2 $2^{-1-}$ mice gain weight similarly to controls and have no defects of brain development or gross adult brain structure, as assessed by serial histochemical staining through the entire brain at closely spaced intervals (data not shown). SHIRPA analysis (Rogers et al., 1997) of wild-type versus knock-out $(\mathrm{KO})$ mice failed to detect any behavioral or physical a P F-Box $\quad$ FBA
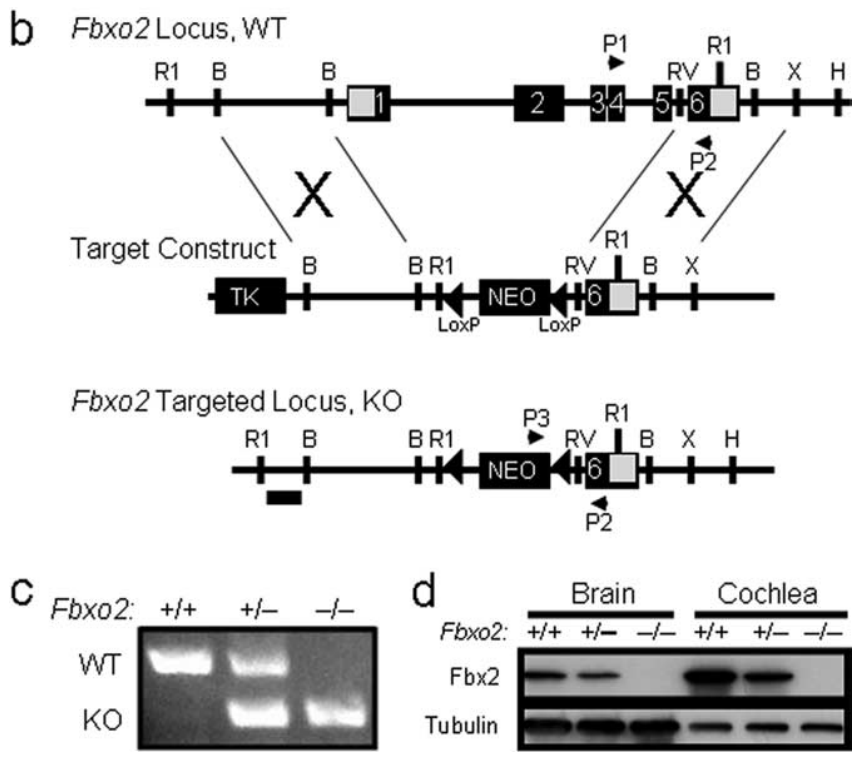

Figure 1. Deletion of mouse Fbxo2.a, Schematic representation of the Fbx2 protein and its functional domains. The F-box binds Skp1, and the FBA domain binds high-mannose glycoprotein substrates. The PEST domain (P) mediates interactions with CHIP (Nelson et al., 2006) and is necessary for Fbx2 to prevent glycoprotein aggregation in vitro (Yoshida et al., 2007). $\boldsymbol{b}$, Knock-out strategy for Fbxo2 using homologous recombination. The targeting construct was designed with thymidine kinase (TK) and neomycin (Neo) resistance cassettes. The Neo cassette is flanked with loxP sites (triangles). Successful homologous recombinants lack the first five exons of $\mathrm{FbxO2}$ and were confirmed by Southern blot analysis using a DNA probe (horizontal bar). Primers P1-P3 were used in PCR genotyping to distinguish WT from KO mice. R1, EcoRI; $B$, BamHl; RV, EcoRV; X, Xhol. c, Representative PCR genotyping of tail DNA for Fbxo2 in WT (+/ $+)$, heterozygotes $(+/-)$, and $\mathrm{KO}(-/-)$ mice using primers P1-P3. $\boldsymbol{d}$, Western blot analysis of Fbx2 expression in brain and cochlea (200 $\mu \mathrm{g}$ of protein in each lane) from wild-type control $\left(\mathrm{FbxO2}^{+/+}\right)$, heterozygous (FbxO2 $\left.2^{+/-}\right)$, and knock-out $\left(\mathrm{Fb} \times 2^{-1-}\right)$ mice. Tubulin is shown as a loading control.

differences except for the auditory function described later in this report.

By Western blot analysis (Fig. 1d), Fbx2 was found to be expressed both in brain and in cochlea, with the expression levels correlating with Fbxo2 gene dosage. In WT mice $\left(\mathrm{Fbxo2}^{+/+}\right)$, cochlear expression of $\mathrm{Fbx} 2$ is at least two times greater per gram of tissue than brain expression of Fbx2 (Fig. 1d). Thus, although Fbx2 has been described as a brain-specific F-box protein (Erhardt et al., 1998), its abundance is even greater in the inner ear. This result is consistent with earlier biochemical studies showing that Fbx2, also known as OCP1, is one of the two most abundant proteins in the inner ear of the guinea pig (Thalmann et al., 1997).

We confirmed by IF that Fbx2 is highly expressed in the cochlea (Fig. 2). Fbx2 is expressed in epithelial support cells (inner sulcus and Claudius' cells) and hair cells of the organ of Corti (Fig. 2a) as well as in the spiral ligament. Fbx2 expression was much less robust in the stria vascularis (Fig. $2 a, b$ ). The expression pattern for Fbx 2 correlates with the published binding pattern of the lectin ConA (Rueda et al., 2003), which, like Fbx2, specifically recognizes high-mannose $\mathrm{N}$-linked glycoproteins. This concordant expression pattern for Fbx2 and ConA-detectable glycoproteins is consistent with a role for Fbx2 in glycoprotein homeostasis in the organ of Corti.

At the subcellular level, however, we did not observe colocalization of Fbx2 with membrane-localized connexin 26, an impor- 
tant inner ear gap junction protein (Fig. $2 b$ ). Connexin 26 was previously shown to interact with Fbx2 in coimmunoprecipitation (Co-IP) experiments (Henzl et al., 2004). Whereas Cx26 displays a patchy distribution consistent with its localization to gap junctions, Fbx2 is diffusely distributed throughout epithelial cells, consistent with its known cytosolic localization. However, it is possible that cytoplasmically localized Cx26 is not detected during immunolabeling and thus fails to colocalize with Fbx2 in this study. In contrast, the known binding partner for Fbx2, Skp1, shows a similar, diffuse cytosolic distribution to Fbx2 (supplemental Fig. 1, available at www.jneurosci.org as supplemental material).

The abundant Fbx2 expression in the inner ear led us to test auditory function in $\mathrm{FbxO2}^{-1-}$ mice. We determined single ABR thresholds on aging, littermatematched cohorts of mice. Fbxo2 $2^{+/+}$and Fbxo2 $2^{-1-}$ mice have similar ABR thresholds and waveforms at 1 month of age (supplemental Fig. 2, available at www. jneurosci.org as supplemental material). However, at 2 months of age, Fbxo $2^{-1-}$ mice begin to develop hearing loss compared with WT mice, as evidenced by the altered ABR waveforms (Fig. $3 a$ ). Hearing loss is evident by $2-4$ months of age (Fig. $3 b$ ), and, by 9 months of age, $\sim 50 \%$ of Fbxo2 $2^{-1-}$ mice have no ABR response. Although WT C57BL/6 mice begin to display hearing loss at 10 months of age because of a well known homozygous mutation in $C d h 23^{\text {ahl }}$ (Noben-Trauth et al., 2003), Fbxo2 ${ }^{-1-}$ mice display much earlier and more profound hearing deficits compared with WT mice.

Consistent with the variability in ABR thresholds at 2-4 months of age, we observed variability in cochlear pathology in young $\mathrm{FbxO2}^{-1-}$ littermates. At 6 weeks of age, hair cells in the organ of Corti of $\mathrm{Fbxo2}^{-1-}$ mice are still present, but there is a loss of supporting cells (Fig. 4). Specifically, the support cells between the outer hair cells and the spiral ligament appear to be lost first. Between 2 and 3.5 months of age, dysmorphic changes are present in the inner sulcus supporting cells, which reside between the inner hair cell and spiral limbus, but the spiral ganglion remains normal. By 5 months, however, both hair cell and spiral ganglion neurodegeneration are seen in $\mathrm{Fbxo}^{-1-}$ mice, whereas wild-type mice do not show signs of degeneration (Fig. 4; supplemental Fig. 3, available at www.jneurosci.org as supplemental material). In contrast, milder degenerative changes are not observed in wild-type mice until $\sim 11$ months of age.

To gain insight into the mechanism of $\mathrm{Fb} x 2$-induced deafness, we studied several proteins implicated in Fbx2 function. We focused our analysis on 2.5-month-old $\mathrm{Fbxo2}^{-1-}$ mice because histologic changes have started to occur by this time, yet the cells of the organ of Corti are still present. As shown previously, the expression patterns of Fbx2 and Skp1 are similar and concordant in the cochlea of wild-type mice (Fig. 5a; supplemental Fig. 1, available at www.jneurosci.org as supplemental material), consistent with their known interaction (Henzl et al., 2001). Intriguingly, staining for Skp1 in Fbxo2 $2^{-1-}$ cochlea was markedly de- creased throughout the length of the cochlea in all Fbxo2 ${ }^{-1-}$ mice that we examined (representative examples are shown in Fig. $5 a$ ). This decrease in cochlear Skp1 was confirmed by Western blot analysis of isolated cochlear tissue lysates (Fig. 5b,c). The marked reduction in Skp1 levels when Fbx2 is absent suggests that most Skp1 activity in the cochlea is functionally associated with Fbx2. In contrast, this does not appear to be the case in brain, in which Skp1 levels remain unchanged in the $\mathrm{Fbxo2}^{-1-}$ brain (Fig. $5 b$ ).

With the possible exception of Cx26 (Henzl et al., 2004), Fbx2 substrate glycoproteins in the cochlea have not yet been identified. Nevertheless, one can gauge total high-mannose glycoprotein levels by immunostaining or immunoblotting with the lectin ConA. Although there is some loss in ConA labeling of the spiral limbus in the cochlea of Fbxo2 $2^{-1-}$ mice (Fig. $5 a$ ), total ConAreactive proteins appeared unchanged in lectin blots of $\mathrm{Fb}_{\mathrm{b}} 2^{-1-}$ cochlea and brain (Fig. 5b). Thus, loss of Fbx2 does not lead to overall global changes in high-mannose glycoproteins in the brain or cochlea. However, expression of ubiquitin and the ER lumenal chaperone, calnexin, were altered in certain areas of the cochlea that normally express Fbx2 [e.g., spiral limbus (Fig. 5a)].

To form a functional ubiquitin ligase, F-box proteins such as Fbx2 typically interact with Skp1 as part of a larger, multisubunit complex known as the SCF complex. SCF complexes also contain the proteins Cullin1 and Rbx1 (Petroski and Deshaies, 2005). If most cochlear Fbx2 resides within SCF complexes, we would expect that Cullin1 and Rbx1 levels would parallel those of Skp1 in Fbxo2 $2^{-1-}$ mice and should decrease with Skp1. However, we did not observe an associated reduction in these other SCF components with which $\mathrm{Fbx} 2$ is presumed to complex (Fig. $5 b$ ). Because a small subset of Skp1/F-box protein ubiquitin ligases may con- 
a
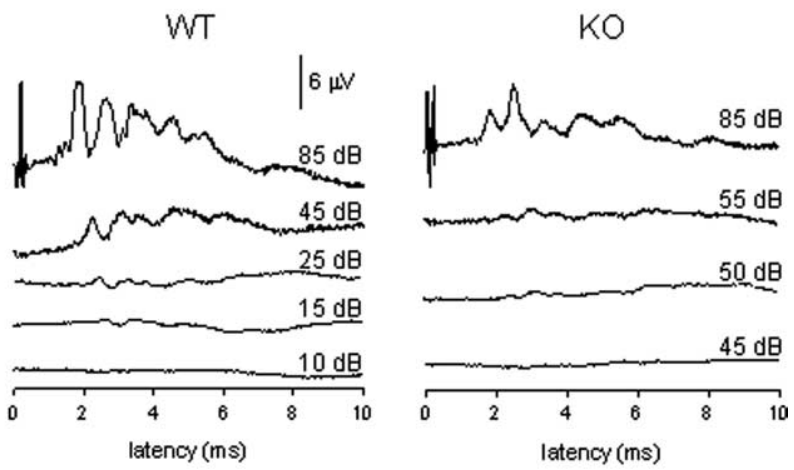

b

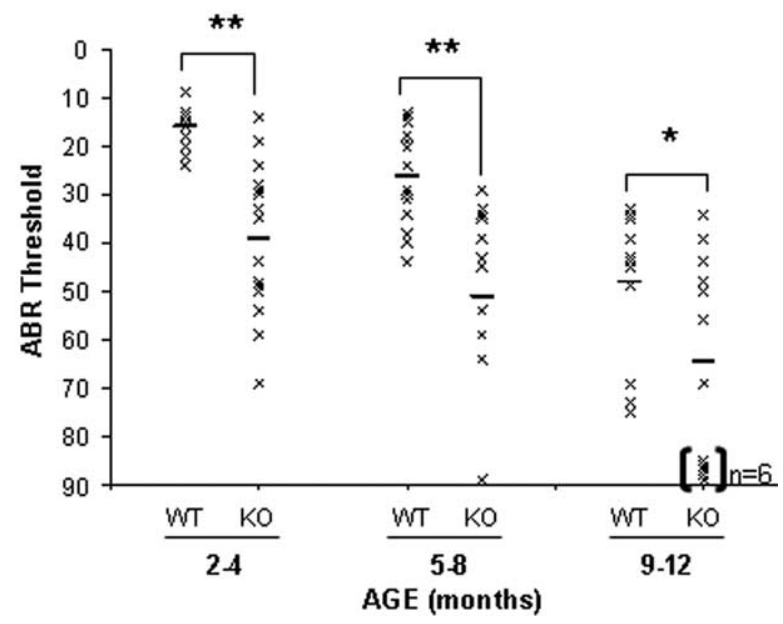

Figure 3. Fbxo2 $2^{-1-}$ mice develop age-related hearing loss. $\boldsymbol{a}$, Representative ABR waveforms from 2-month-old Fbxo2 WT and KO mice, with thresholds of 15 and $50 \mathrm{~dB}$, respectively. $\boldsymbol{b}$, ABR thresholds of aging, littermate-matched cohorts for WT and FbxO2 $2^{-/-}$(KO) mice. ABR thresholds for each mouse (crosses) and the group mean (horizontal bars) are designated. Square brackets and " $n=6$ " indicate that six mice in the 9- to 12-month-old group have no ABR response and were classified as deaf. Statistical significance was determined from the mean \pm SD $\left({ }^{*} p<0.01 ;{ }^{* *} p<0.001\right)$.

tain a different cullin, Cullin7, we also assessed Cullin7 levels in WT versus $\mathrm{Fbxo}^{-1-}$ mice. In Fbxo2 $2^{-1-}$ brain and cochlea, Cullin7 levels also remained essentially unchanged at 1.5 and 4.5 months of age (Fig. $5 d$ ).

These results led us to consider the possibility that, in the cochlea at least, Fbx2 interacts with Skp1 as a heterodimer yet does not form a conventional SCF complex. To assess this possibility, we analyzed the interaction of Fbx2 and Skp1 in Co-IP assays from cochlear and brain extracts. The results showed that Skp1 and Fbx2 do coprecipitate from cochlear and brain lysates (Fig. 6a,b, respectively), suggesting a direct interaction between these two proteins in vivo, as expected based on earlier studies (Yoshida et al., 2002; Henzl et al., 2004). We also confirmed the interaction of $\mathrm{Fbx} 2$ with a recently identified substrate glycoprotein in brain, the NR1 subunit of the NMDA receptor (Fig. 6b) (Kato et al., 2005).

Although results by others have supported the formation of full SCF complexes when Fbx2 is coexpressed with other subunits of SCF complexes in transfected cells (Yoshida et al., 2002; Murai-Takebe et al., 2004), we consistently failed to observe coprecipitation of Cullin 1 and Rbx1 with endogenous Fbx2 in cochlear and brain extracts (Fig. 6a,b). This absence of coprecipitation occurred even under the mildest conditions of detergent extraction ( $0.5 \% \mathrm{NP}-40$ in a low salt solution). We observe little, if any, coprecipitation of Cullin1 and Rbx1 with Fbx2 in cells overexpressing Fbx2 and all other SCF subunits (Fig. $6 c, d$ ). This weak coprecipitation of Rbx1 and Cullin 1 with Fbx2 is much less than the amount that coprecipitates with related F-box proteins from the Fbx2 family. For example, the F-box protein most similar to Fbx2, Fbx6, readily coprecipitates with Cullin1 and Skp1, whereas Fbx2 coprecipitates only with Skp1 (Fig. 6c,d). These results, together with our finding that Cullin 1 and Rbx1 levels are unaltered in the Fbxo2 ${ }^{-1-}$ cochlea, whereas Skp1 levels are reduced (Fig. 2b), suggest that Fbx2 and Skp1 function in the cochlea in a unique protein complex distinct from conventional SCF complexes. Interestingly, other laboratories also recently failed to show coprecipitation of appreciable quantities of Cullin1 protein with endogenous Fbx2 (Yoshida et al., 2002, 2007; Tai, 2006).

Fbx2 has been shown by others to bind Cx26 (Henzl et al., 2004), a gap junction protein that is critically important to cochlear homeostasis. Mutations in Cx26 cause nearly one-half of all cases of recessive, nonsyndromic deafness (Kelsell et al., 1997). In similar coprecipitation experiments, we were unable to confirm a direct interaction between Fbx2 and Cx26 in cochlear lysates or in transfected cells (data not shown). Because Cx26 lacks high-mannose glycans, the known binding target of Fbx2 (Yoshida et al., 2002), this result is perhaps not surprising. In $\mathrm{Fbxo2} 2^{-1-}$ mice, however, we did observe increased levels of Cx26 in a subset of 2.5-month-old mice (Fig. 7) (ratio of KO to WT $=$ $2.23 \pm 0.36$; mean \pm SEM; $n=3 ; p<0.01)$. However, levels of another gap junction protein expressed in the inner ear, connexin 30, were not changed (Fig. 7) (ratio of $\mathrm{KO}$ to $\mathrm{WT}=0.85 \pm 0.12$; mean \pm SEM; $n=3$; not significant). This finding suggests that Fbx2 assists in regulating Cx26 levels, regardless of whether this is mediated by a direct interaction between the two proteins.

To identify changes in Fbxo2 $2^{-1-}$ cochlea at the ultrastructural level, we performed TEM on cochlea from 2- and 3.5-month-old $\mathrm{Fbxo2}^{-1-}$ mutants. This revealed abnormal juxtamembranous, tubulovesicular structures in hair cells and the Claudius' cells of the organ of Corti in Fbxo2 $2^{-1-}$ mice (Fig. 8). These structures appear partially contiguous with the plasmalemma and accumulate asymmetrically on one side of two abutting cell membranes (Fig. 8a,b). These juxtamembranous structures, which were present at cell membrane junctions of Claudius' cells, may reflect alterations in connexon/gap junction biology. Gap junctions have been noted to have a rapid turnover rate of $1-5 \mathrm{~h}$ (Berthoud et al., 2004). Electron microscopy and antibody labeling studies have shown that gap junctions undergo asymmetric endocytosis to form annular gap junctions (Jordan et al., 2001). It is therefore possible that the juxtamembranous structures in $\mathrm{Fbxo2}^{-/-}$cells include endocytosed connexins that fail to be degraded by an Fbx2-dependent pathway.

\section{Discussion}

We have shown that deletion of Fbxo2 leads to accelerated, agerelated hearing loss with cochlear degeneration in mice. Degeneration is restricted to the cochlea despite Fbx2 expression in brain, implying that Fbx2 plays a uniquely important role in cochlear homeostasis. We also present in vivo evidence to suggest that Fbx2 and Skp1 form a heterodimer rather than a classic, tetrameric SCF complex in the inner ear. Given the known substrate specificity of Fbx2 for high-mannose-containing glycoproteins, and the presence of membranous accumulations in the cochlea of $\mathrm{Fbxo2}^{-1-}$ mice, we propose that $\mathrm{Fbx} 2$ participates in 
cochlear membrane glycoprotein homeostasis through a novel cellular pathway.

Previously, Thalmann et al. (1997) showed that Fbx2 (OCP1) and Skp1 (OCP2) are the most abundant proteins in the organ of Corti, each comprising 5\% of protein in this tissue in the guinea pig. This abundance is remarkably high for a regulatory protein such as a ubiquitin ligase. It raises the intriguing possibility that in the organ of Corti, Fbx2 and Skp1 act in a noncanonical manner (that is, not as a traditional SCF ubiquitin ligase). Together with earlier in vitro studies (Henzl et al., 2004) and recent cell-based studies (Yoshida et al., 2007), our results are consistent with this view. Fbx2 and Skp1 strongly and directly interact and Skp1 levels are significantly reduced in the cochlea of $\mathrm{Fbxo2}^{-1-}$ mice. However, unlike other related F-box proteins (e.g., Fbx6), Fbx2 cannot coprecipitate other components of the SCF complex, Cullin1 and Rbxl. In agreement with this observation, Cullin 1 and Rbx1 levels are not decreased in Fbxo2 $2^{-1-}$ mice cochleae. Thus, our data suggest that Skp1 and Fbx2 form an atypical heterodimer in the cochlea rather than a traditional SCF tetramer.

It is possible that Skp1/Fbx2 heterodimers could interact with other Cullin homologues that are known to interact with Skp1. Cullin7, for example, forms an SCF ubiquitin ligase complex with Fbx29 (Dias et al., 2002; Huber et al., 2005) and is expressed in the organ of Corti (Fig. 5b). However, Cullin7 levels were not reduced in the cochlea of $\mathrm{Fbxo2}^{-1-}$ mice, suggesting that Fbx2 and Skp1 do not function with Cullin7.

Emerging evidence suggests that not all F-box proteins necessarily form classic, tetrameric SCF complexes. For example, the yeast F-box protein RCY1 binds Skp1 yet fails to form an SCF complex and functions to recycle internalized proteins ( $\mathrm{Ga}$ lan et al., 2001). Like RCY1, Fbx2 may have a nontraditional, Skp1-dependent function in vivo to regulate membrane protein turnover that is distinct from its predicted function as a ubiquitin ligase. Other than its F-box domain, RCY1 has no sequence similarity to Fbx2. Importantly, our model of a stable Skp1/Fbx2 heterodimer does not rule out a possible dynamic, or regulated, association of this complex with Cullin1 and Rbx1, or with other conjugation machinery, to mediate ubiquitination of bound substrates. In the absence of such machinery, the Skp1/Fbx 2 complex would lack the capacity to ubiquitinate substrates. One possibility is that the Skp1/Fbx2 heterodimer has unique functions independent of its action as a ubiquitin ligase. Indeed, as we were finishing the prep-

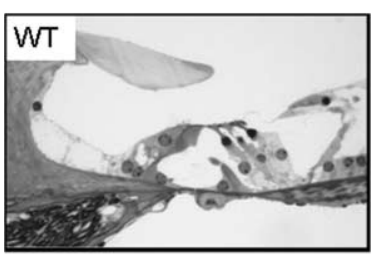

5 Month
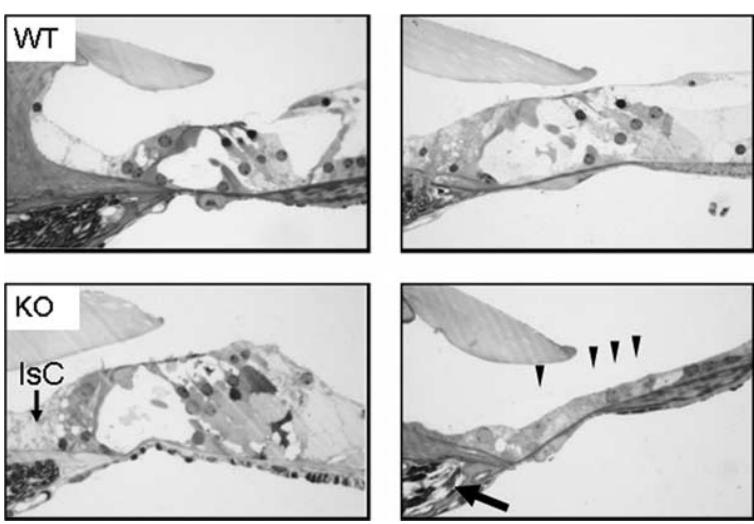

11 Month
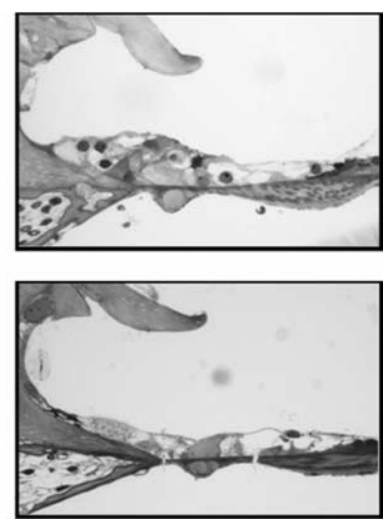

Figure 4. Fbxo2 $2^{-1-}$ mice develop age-related cochlear degeneration. Hematoxylin and eosin staining of representative midmodiolus cochlear sections from WT and $\mathrm{FbxO}^{-/-}$(KO) mice at 1.5, 5, and 11 months of age. Cellular changes begin between 1 and 3.5 months. Support cell loss and dysmorphic changes to inner sulcus cells (IsC) are evident at 1-3 months of age, followed by hair cell (four arrowheads) and neuronal degeneration in the spiral ganglion (arrow) at 3-5 months of age. Note that WT C57BL/6 mice have age-related degeneration at 11 months that is consistent with audiometric data (see Fig. 3 ). a

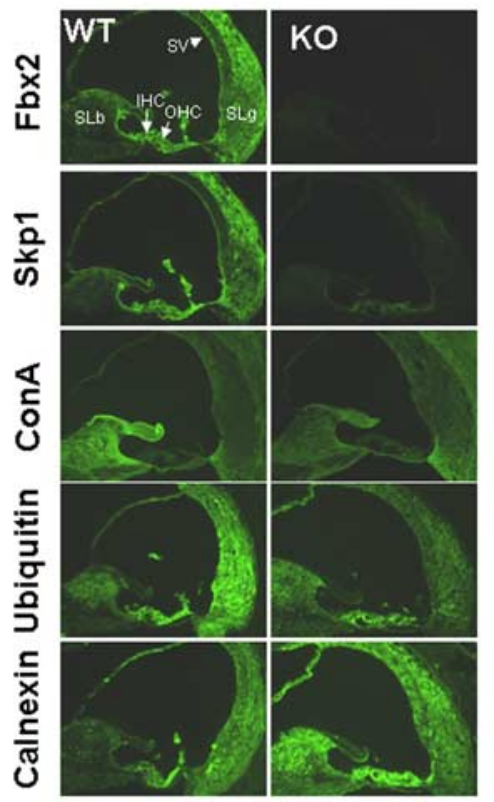

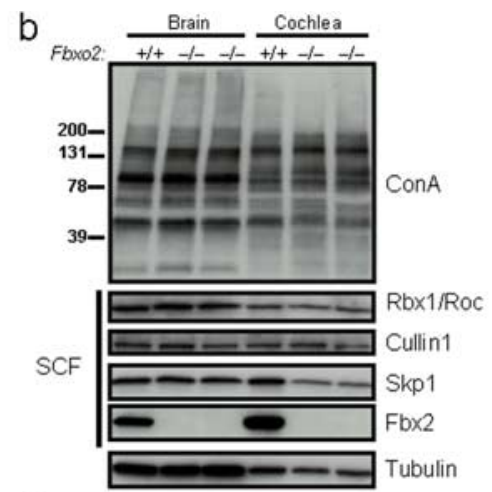

C

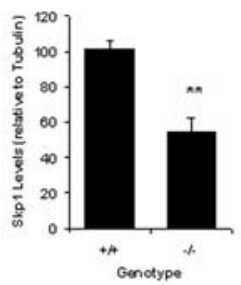

d

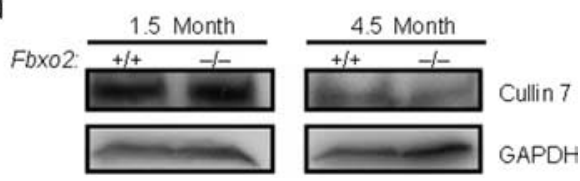

Figure 5. Fbx2 expression regulates Skp1 levels in the cochlea. $\boldsymbol{a}$, Fluorescence microscopy of cochlea from 2-month-old WT and $\mathrm{KO}$ mice stained for the indicated antigens. Fbx2 is absent and Skp1 is markedly decreased in FbxO2 ${ }^{-1-}$ (KO) mice. Levels of ConA-detectable glycoproteins and ubiquitin appear lower in select regions, whereas levels of calnexin were increased in the spiral limbus of KO cochlea. IHC, Inner hair cells; OHC, outer hair cells; SLb, spiral limbus; SLg, spiral ligament; SV, stria vascularis. $\boldsymbol{b}$, Western blot analysis of brain and cochlear lysates (40 $\mu \mathrm{g}$ of each) from 2-month-old WT and K0 mice probed with antibodies specific for the indicated proteins. Results show decreased levels of Skp1, whereas levels of ConA-detectable glycoproteins, Cullin1, and Rbx1/Roc remain unchanged. c, Quantification of cochlear Skp1 levels relative to tubulin $\left(n=3 ;{ }^{* *} p<0.001\right)$. $\boldsymbol{d}$, Western blot analysis of cochlear lysates (40 $\mu \mathrm{g}$ of each) from 1.5- and 4.5-month-old WT and KO mice. Levels of Cullin7 (another cullin expressed in the cochlea) are unchanged in $\mathrm{KO}$ mice.

aration of this manuscript, Yoshida et al. (2007) reported cellbased studies showing that Fbx2/Skp1 heterodimers may act to prevent glycoprotein aggregation.

Regardless of the mode of Fbx2 action in the cochlea, previous work has shown that Fbx2 binds and ubiquitinates misfolded, high-mannose-containing membrane glycoproteins (Yoshida et 
a
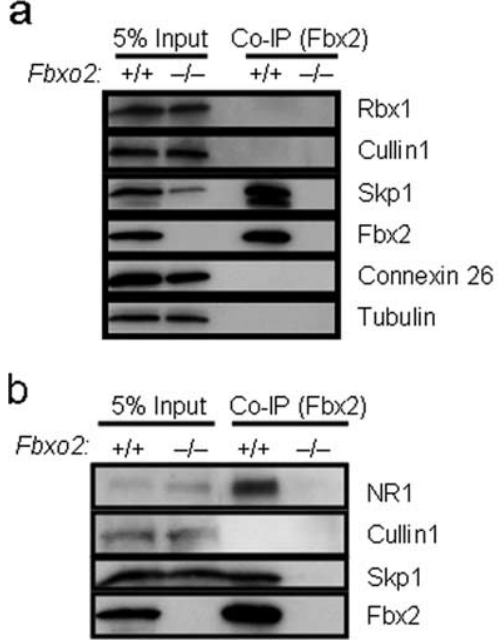

C

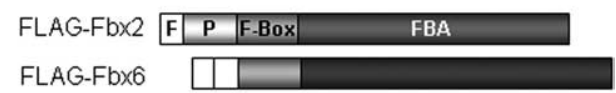

d

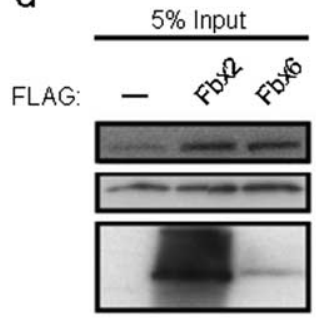

Co-IP (FLAG)

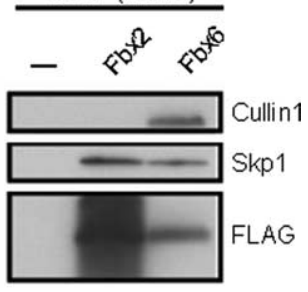

Figure 6. Coprecipitation of Skp1, but not other SCF proteins, with Fbx2. $\boldsymbol{a}, \boldsymbol{b}$, Co-IP of endogenous Fbx2 and Skp 1 from cochlear $(\boldsymbol{a})$ and brain $(\boldsymbol{b})$ extracts from 2-month-old wild-type $\left(\mathrm{FbxO2}^{+/+}\right)$and knock-out $\left(\mathrm{FbxO2} 2^{-/-}\right)$mice. Other SCF components (Cullin1 and Rbx1) did not coprecipitate with Fbx2 from lysates. c, Schematic diagram of FLAG epitope-tagged Fbx2 and Fbx6 constructs used to transfect Cos cells in $\boldsymbol{d}$. F, FLAG; P, PEST. d, Co-IP of FLAG-tagged proteins coexpressed in Cos cells with Skp1, Cullin1, and Rbx1. Note that Fbx6 coprecipitates Skp1 and Cullin1, whereas Fbx2 only coprecipitates Skp1. Input indicates 5\% of the total starting lysates subjected to Co-IP. — , Overexpression of Skp1, Cullin1, and Rbx1 and empty vector substitution for Fbx2 and Fbx6.

\subsection{Month Cochlea}

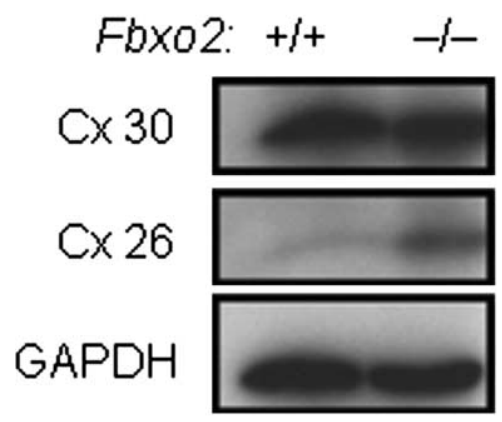

Figure 7. Connexin 26, but not connexin 30, levels are increased in $\mathrm{FbxO2}^{-1-}$ mice cochlea. Representative Western blot of cochlear extracts from 2.5-month-old wild-type $\left(\mathrm{FbxO2}^{+/+}\right)$ and knock-out $\left(\mathrm{FbxO2} 2^{-/-}\right.$) mice. Note increased levels of $\mathrm{C} \times 26$, but not $\mathrm{C} \times 30$, in knock-out mice. Relative levels of Cx26 and Cx30 cannot be compared directly by this Western blot approach.

al., 2002; Murai-Takebe et al., 2004; Hagihara et al., 2005; Kato et al., 2005; Nelson et al., 2006). We propose that the loss of hearing observed in $\mathrm{Fbxo2}^{-/-}$mice is caused by a failure of glycoprotein quality control in the organ of Corti. These substrate glycoproteins may derive from the endoplasmic reticulum through ERassociated degradation or from the cell surface through an as-yet-unknown mechanism. A small number of cell-surface glycoproteins retain their high-mannose glycan status, and these now represent candidate substrates for Fbx2 action. Unfortunately, it is currently unknown which cell-surface glycoproteins in the cochlea retain high-mannose glycans. On ConA blots, we failed to observe obvious alterations in levels of high-mannose glycoproteins in the cochlea of $\mathrm{Fbxo2}^{-/-}$mice. A focused investigation on predicted candidate substrates will be required to detect alterations in specific, low-abundance glycoprotein substrates.

Mouse models and human genetic disorders have been invaluable in aiding our understanding of normal cochlear func- tion and its dysfunction in deafness. Approximately one-half of all forms of inherited deafness result from autosomal recessive loss of $\mathrm{Cx} 26$, one of four connexins (connexins 26, 30,31, and 43) expressed in the inner ear. Mice conditionally deleted of Cx26 (Cohen-Salmon et al., 2002), deficient in Cx30 (Teubner et al., 2003), or expressing mutant potassium channels (Kubisch et al., 1999; Lee et al., 2000) all display hearing impairment. These effects highlight the importance to cochlear homeostasis of controlling ion fluxes.

Cx26 is a potential target for Fbx2, because it has been shown to coprecipitate with Fbx2 (Henzl et al., 2004). In support of this, we found increased levels of Cx26 in a subset of $\mathrm{Fbxo2}^{-I-}$ mice before cellular degeneration was widespread. However, Cx26 contains no plausible N-linked glycosylation sites, and there is no published evidence supporting $\mathrm{N}$ - or O-linked glycosylation of Cx26. Therefore, Cx26 and other connexins are not obvious candidates to bind Fbx2 directly. How do we reconcile this with previous work showing that $\mathrm{Fbx} 2$ coprecipitates with $\mathrm{Cx} 26$ in guinea pig cochlea (Henzl et al., 2004)? Coprecipitation does not prove a direct physical connection and could reflect, instead, an indirect interaction via one or more additional proteins. Additional in vitro studies showing a direct interaction between $\mathrm{Fbx} 2$ and $\mathrm{Cx} 26$ (Henzl et al., 2004) may have been complicated by the fact that in vitro translated membrane proteins could interact nonspecifically with other proteins when expressed in the absence of microsomal membranes. In contrast, we have been unable to detect an interaction between Fbx2 and Cx26 in mouse cochlea (Fig. 6a) or in transfected cells (data not shown). Sequence variations in Fbx2 from guinea pig and mouse likely do not account for these observed differences in Cx26 binding, because they are 82\% identical. Based on the established substrate specificity of Fbx2 for glycoproteins and the findings presented here, we speculate that the increased Cx26 level observed in Fbxo2 ${ }^{-1-}$ mice reflects a broader problem in membrane protein turnover rather than a specific effect on Cx26.

The brain and neurological behavior of $\mathrm{Fb}_{00} 2^{-1-}$ mice appear normal despite the robust expression of $\mathrm{Fbx} 2$ throughout the CNS. Moreover, the fact that Skp1 levels in the brain do not decrease when $\mathrm{Fbx} 2$ is missing suggests that, in contrast to the situation in the cochlea, most Skp1 in the brain is not functionally linked to Fbx2. Indeed, many other F-box proteins are expressed in brain. We speculate that in the brain most Skp1 resides in SCF complexes that do not contain Fbx2. These data, however, do not rule out an important function for $\mathrm{Fbx} 2$ in the brain. Additional studies in brain are needed to determine whether levels of specific glycoproteins are altered in Fbx2 brain and whether subtle changes in neuronal function occur when Fbx2 is deleted.

Based on our data, FBXO2, which maps to human chromosome 1 in band p36 (supplemental Fig. 4, available at www.jneurosci.org as supplemental material), becomes a candidate genetic modifier in age-related hearing loss in humans. Several groups of patients have hearing loss related to genetic changes in 1p36. In fact, $F B X O 2$ resides within the large 6 centimorgan DFNB36 loci believed to be caused by mutations in ESPN 
(Naz et al., 2004). In contrast, FBXO2 is not located within the $1 \mathrm{p} 36$ deletion region known to cause developmental abnormalities and hearing loss (Shapira et al., 1997; Heilstedt et al., 2003). Furthermore, the significant decrease in Skp1 levels in the Fbxo2 $2^{-I-}$ mice suggests that SKP1A, located at human chromosome 5 in band q31 (supplemental Fig. 4, available at www.jneurosci.org as supplemental material), likely plays a role in auditory function. With the recent publication of the human HapMap (Altshuler et al., 2005), genetic association studies can begin for known FBXO2 small nucleotide polymorphisms (SNPs). There are currently two identified SNPs for FBXO2 [K118T and R121S (supplemental Fig. 5, available at www.jneurosci.org as supplemental material)]. Our studies failed to show a significant correlation between age-related hearing impairment in affected individuals $(n=534)$ and with K118T SNP in FBXO2 (R. F. Nelson, R. J. H. Smith, and G. Van Camp, unpublished observations). We also plan to investigate the functional relevance of the R121S SNP and to investigate FBXO2 genetic mutations in recessive and agerelated human deafness.

These findings constitute the first demonstration of a direct link between a protein implicated in ubiquitin pathways and hearing loss. The cochlear degeneration that occurs in mice null for Fbxo2 suggests that ongoing protein quality control is essential to the health of the inner ear. Important issues that now can be addressed include identifying substrate glycoproteins and membrane proteins that are regulated by Fbx2, determining how Fbx2 and Skp1 function in an apparently novel protein complex, and identifying the mechanism of cell death in the cochlea. A very recent study suggests that Fbx2/Skp1 may also function as a chaperone to prevent glycoprotein aggregation and that this function depends on the N-terminal PEST domain of Fbx2 (Yoshida et al., 2007). Consistent with these data, Fbx2 interacts with the cochaperone/ubiquitin ligase $\mathrm{C}$ terminus of Hsc70-interacting protein (CHIP) through the PEST domain (Nelson et al., 2006). Future experiments should also address auditory phenotypes in mice null for CHIP and in Fbxo2/CHIP double knock-out mice. Finally, it will be important to determine more globally whether protein turnover and ubiquitin pathways are required for cochlear homeostasis and, if so, whether these pathways can be therapeutically modified to delay or prevent age-related hearing loss.

\section{References}

Altshuler D, Brooks LD, Chakravarti A, Collins FS, Daly MJ, Donnelly P (2005) A haplotype map of the human genome. Nature 437:1299-1320.

Beltramello M, Piazza V, Bukauskas FF, Pozzan T, Mammano F (2005) Impaired permeability to Ins $(1,4,5) \mathrm{P} 3$ in a mutant connexin underlies recessive hereditary deafness. Nat Cell Biol 7:63-69.

Berthoud VM, Minogue PJ, Laing JG, Beyer EC (2004) Pathways for degradation of connexins and gap junctions. Cardiovasc Res 62:256-267.

Cohen-Salmon M, Ott T, Michel V, Hardelin JP, Perfettini I, Eybalin M, Wu T, Marcus DC, Wangemann P, Willecke K, Petit C (2002) Targeted ab-

\section{2 mo}

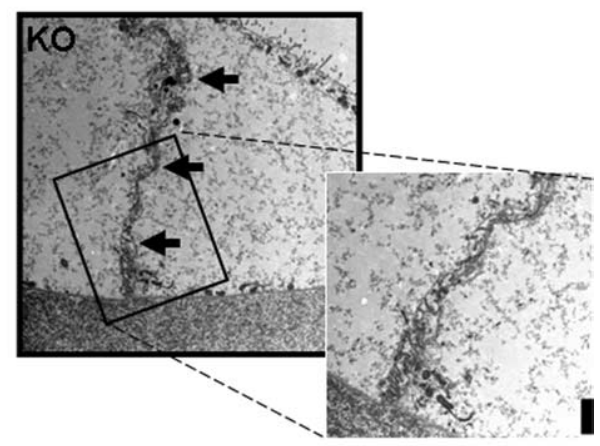

$3.5 \mathrm{mo}$

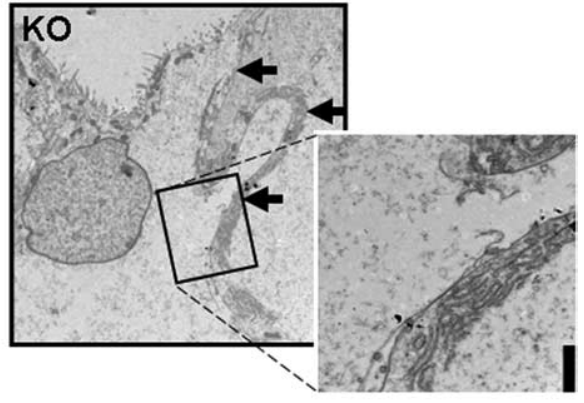

3.5

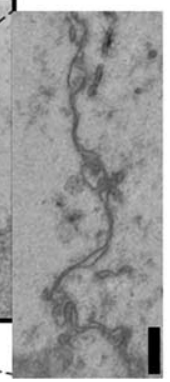

in suppo

support cells of the cochlea. $\boldsymbol{a}, \boldsymbol{b}$, TEM analysis of cochlea from 2-month-old (2 mo; $\boldsymbol{a})$ and 3.5-month-old $(3.5 \mathrm{mo} ; \boldsymbol{b})$ WT and KO mice showing disruption of support cell (Claudius' cells) membrane integrity (black arrows). Inset photo, An asymmetric, juxtamembranous accumulation of membranous structures is mice at 3.5 months. Scale bars: $2 \mu \mathrm{m}$; insets, $1 \mu \mathrm{m}$.

lation of connexin26 in the inner ear epithelial gap junction network causes hearing impairment and cell death. Curr Biol 12:1106-1111.

Corey DP, Garcia-Anoveros J, Holt JR, Kwan KY, Lin SY, Vollrath MA, Amalfitano A, Cheung EL, Derfler BH, Duggan A, Geleoc GS, Gray PA, Hoffman MP, Rehm HL, Tamasauskas D, Zhang DS (2004) TRPA1 is a candidate for the mechanosensitive transduction channel of vertebrate hair cells. Nature 432:723-730.

Dias DC, Dolios G, Wang R, Pan ZQ (2002) CUL7: A DOC domaincontaining cullin selectively binds Skp1.Fbx29 to form an SCF-like complex. Proc Natl Acad Sci USA 99:16601-16606.

Erhardt JA, Hynicka W, DiBenedetto A, Shen N, Stone N, Paulson H, Pittman RN (1998) A novel F box protein, NFB42, is highly enriched in neurons and induces growth arrest. J Biol Chem 273:35222-35227.

Galan JM, Wiederkehr A, Seol JH, Haguenauer-Tsapis R, Deshaies RJ, Riezman H, Peter M (2001) Skp1p and the F-box protein Rcylp form a non-SCF complex involved in recycling of the SNARE Snclp in yeast. Mol Cell Biol 21:3105-3117.

Guan X, Ruch RJ (1996) Gap junction endocytosis and lysosomal degradation of connexin43-P2 in WB-F344 rat liver epithelial cells treated with DDT and lindane. Carcinogenesis 17:1791-1798.

Hagihara S, Totani K, Matsuo I, Ito Y (2005) Thermodynamic analysis of interactions between $\mathrm{N}$-linked sugar chains and F-box protein Fbs1. J Med Chem 48:3126-3129.

Heilstedt HA, Ballif BC, Howard LA, Lewis RA, Stal S, Kashork CD, Bacino CA, Shapira SK, Shaffer LG (2003) Physical map of 1p36, placement of breakpoints in monosomy $1 \mathrm{p} 36$, and clinical characterization of the syndrome. Am J Hum Genet 72:1200-1212.

Helenius A, Aebi M (2004) Roles of N-linked glycans in the endoplasmic reticulum. Annu Rev Biochem 73:1019-1049.

Henzl MT, O’Neal J, Killick R, Thalmann I, Thalmann R (2001) OCP1, an F-box protein, co-localizes with OCP2/SKP1 in the cochlear epithelial gap junction region. Hear Res 157:100-111.

Henzl MT, Thalmann I, Larson JD, Ignatova EG, Thalmann R (2004) The cochlear F-box protein OCP1 associates with OCP2 and connexin 26. Hear Res 191:101-109.

Huber C, Dias-Santagata D, Glaser A, O’Sullivan J, Brauner R, Wu K, Xu X, Pearce K, Wang R, Uzielli ML, Dagoneau N, Chemaitilly W, SupertiFurga A, Dos Santos H, Megarbane A, Morin G, Gillessen-Kaesbach G, 
Hennekam R, Van der Burgt I, Black GC, et al. (2005) Identification of mutations in CUL7 in 3-M syndrome. Nat Genet 37:1119-1124.

Jentsch TJ (2000) Neuronal KCNQ potassium channels: physiology and role in disease. Nat Rev Neurosci 1:21-30.

Jordan K, Chodock R, Hand AR, Laird DW (2001) The origin of annular junctions: a mechanism of gap junction internalization. J Cell Sci 114:763-773.

Kato A, Rouach N, Nicoll RA, Bredt DS (2005) Activity-dependent NMDA receptor degradation mediated by retrotranslocation and ubiquitination. Proc Natl Acad Sci USA 102:5600-5605.

Kelsell DP, Dunlop J, Stevens HP, Lench NJ, Liang JN, Parry G, Mueller RF, Leigh IM (1997) Connexin 26 mutations in hereditary non-syndromic sensorineural deafness. Nature 387:80-83.

Kubisch C, Schroeder BC, Friedrich T, Lutjohann B, El-Amraoui A, Marlin S, Petit C, Jentsch TJ (1999) KCNQ4, a novel potassium channel expressed in sensory outer hair cells, is mutated in dominant deafness. Cell 96:437-446.

Laing JG, Beyer EC (1995) The gap junction protein connexin 43 is degraded via the ubiquitin proteasome pathway. J Biol Chem 270:26399-26403.

Lee MP, Ravenel JD, Hu RJ, Lustig LR, Tomaselli G, Berger RD, Brandenburg SA, Litzi TJ, Bunton TE, Limb C, Francis H, Gorelikow M, Gu H, Washington K, Argani P, Goldenring JR, Coffey RJ, Feinberg AP (2000) Targeted disruption of the Kvlqtl gene causes deafness and gastric hyperplasia in mice. J Clin Invest 106:1447-1455.

Meusser B, Hirsch C, Jarosch E, Sommer T (2005) ERAD: the long road to destruction. Nat Cell Biol 7:766-772.

Michel JJ, Xiong Y (1998) Human CUL-1, but not other cullin family members, selectively interacts with SKP1 to form a complex with SKP2 and cyclin A. Cell Growth Differ 9:435-449.

Murai-Takebe R, Noguchi T, Ogura T, Mikami T, Yanagi K, Inagaki K, Ohnishi H, Matozaki T, Kasuga M (2004) Ubiquitination-mediated regulation of biosynthesis of the adhesion receptor SHPS- 1 in response to endoplasmic reticulum stress. J Biol Chem 279:11616-11625.

Naz S, Griffith AJ, Riazuddin S, Hampton LL, Battey Jr JF, Khan SN, Wilcox ER, Friedman TB (2004) Mutations of ESPN cause autosomal recessive deafness and vestibular dysfunction. J Med Genet 41:591-595.

Nelson RF, Glenn KA, Miller VM, Wen H, Paulson HL (2006) A novel route for F-box protein-mediated ubiquitination links CHIP to glycoprotein quality control. J Biol Chem 281:20242-20251.

Noben-Trauth K, Zheng QY, Johnson KR (2003) Association of cadherin 23 with polygenic inheritance and genetic modification of sensorineural hearing loss. Nat Genet 35:21-23.

Petroski MD, Deshaies RJ (2005) Function and regulation of cullin-RING ubiquitin ligases. Nat Rev Mol Cell Biol 6:9-20.

Rogers DC, Fisher EM, Brown SD, Peters J, Hunter AJ, Martin JE (1997) Behavioral and functional analysis of mouse phenotype: SHIRPA, a proposed protocol for comprehensive phenotype assessment. Mamm Genome 8:711-713.

Rueda J, Cantos R, Lim DJ (2003) Distribution of glycoconjugates during cochlea development in mice: light microscopic lectin study. Anat Rec A Discov Mol Cell Evol Biol 274:923-933.

Schroder M, Kaufman RJ (2005) ER stress and the unfolded protein response. Mutat Res 569:29-63.

Shapira SK, McCaskill C, Northrup H, Spikes AS, Elder FF, Sutton VR, Korenberg JR, Greenberg F, Shaffer LG (1997) Chromosome 1p36 deletions: the clinical phenotype and molecular characterization of a common newly delineated syndrome. Am J Hum Genet 61:642-650.

Sitia R, Braakman I (2003) Quality control in the endoplasmic reticulum protein factory. Nature 426:891-894.

Tai T (2006) Identification of N-glycan-binding proteins for E3 ubiquitin ligases. Methods Enzymol 415:20-30.

Teubner B, Michel V, Pesch J, Lautermann J, Cohen-Salmon M, Sohl G, Jahnke K, Winterhager E, Herberhold C, Hardelin JP, Petit C, Willecke K (2003) Connexin30 (Gjb6)-deficiency causes severe hearing impairment and lack of endocochlear potential. Hum Mol Genet 12:13-21.

Thalmann R, Henzl MT, Thalmann I (1997) Specific proteins of the organ of Corti. Acta Otolaryngol 117:265-268.

Williamson RA, Henry MD, Daniels KJ, Hrstka RF, Lee JC, Sunada Y, Ibraghimov-Beskrovnaya O, Campbell KP (1997) Dystroglycan is essential for early embryonic development: disruption of Reichert's membrane in Dag1-null mice. Hum Mol Genet 6:831-841.

Yoshida Y, Chiba T, Tokunaga F, Kawasaki H, Iwai K, Suzuki T, Ito Y, Matsuoka K, Yoshida M, Tanaka K, Tai T (2002) E3 ubiquitin ligase that recognizes sugar chains. Nature 418:438-442.

Yoshida Y, Tokunaga F, Chiba T, Iwai K, Tanaka K, Tai T (2003) Fbs2 is a new member of the E3 ubiquitin ligase family that recognizes sugar chains. J Biol Chem 278:43877-43884.

Yoshida Y, Murakami A, Iwai K, Tanaka K (2007) A neural-specific F-box protein Fbs1 functions as a chaperone suppressing glycoprotein aggregation. J Biol Chem 282:7137-7144. 